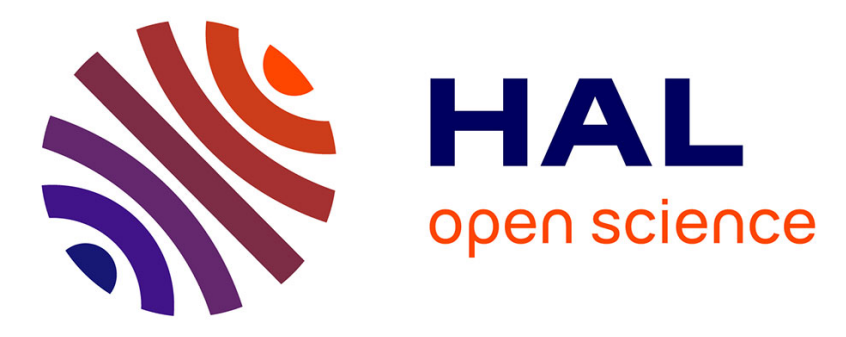

\title{
Automatic tracking of free-flying insects using a cable-driven robot
}

Rémi Pannequin, Melanie Jouaiti, Mohamed Boutayeb, Philippe Lucas, Dominique Martinez

\section{To cite this version:}

Rémi Pannequin, Melanie Jouaiti, Mohamed Boutayeb, Philippe Lucas, Dominique Martinez. Automatic tracking of free-flying insects using a cable-driven robot. Science Robotics, 2020, 5 (43), pp.eabb2890. 10.1126/scirobotics.abb2890 . hal-02891766

\section{HAL Id: hal-02891766 https://hal.science/hal-02891766}

Submitted on 28 Jul 2020

HAL is a multi-disciplinary open access archive for the deposit and dissemination of scientific research documents, whether they are published or not. The documents may come from teaching and research institutions in France or abroad, or from public or private research centers.
L'archive ouverte pluridisciplinaire HAL, est destinée au dépôt et à la diffusion de documents scientifiques de niveau recherche, publiés ou non, émanant des établissements d'enseignement et de recherche français ou étrangers, des laboratoires publics ou privés. 


\title{
Automatic tracking of free-flying insects using a cable-driven robot
}

\author{
Rémi Pannequin ${ }^{1}$, Mélanie Jouaiti ${ }^{2}$, Mohamed Boutayeb ${ }^{1}$, \\ Philippe Lucas ${ }^{3}$, and Dominique Martinez ${ }^{2, *}$ \\ ${ }^{1}$ Université de Lorraine, CNRS, CRAN, 54000 Nancy, France \\ ${ }^{2}$ Université de Lorraine, CNRS, LORIA, 54000 Nancy, France \\ ${ }^{3}$ Institute of Ecology and Environmental Sciences, INRA, 78000 Versailles, France
}

*To whom correspondence should be addressed; E-mail: Dominique.Martinez@loria.fr

Flying insects have evolved to develop efficient strategies to navigate in natural environments. Yet, studying them experimentally is difficult because of their small size and high speed of motion. Consequently, previous studies were limited to tethered flights, hovering flights or restricted flights within confined laboratory chambers. Here, we report the development of a cable-driven parallel robot, named lab-on-cables, for tracking and interacting with a free-flying insect. In this approach, cameras are mounted on cables, so as to move automatically with the insect. We design a reactive controller that minimizes the online tracking error between the position of the flying insect, provided by an embedded stereo-vision system and the position of the moving lab, computed from the cable lengths. We validate the lab-on-cables with Agrotis ipsilon moths (c.a. 2 centimeters long) flying freely up to 3 meters per second. We further demonstrate, using pre-recorded trajectories, the possibility to track other in- 
sects such as fruit flies or mosquitoes. The lab-on-cables is relevant to freeflight studies and may be used in combination with stimulus delivery to assess sensory modulation of flight behavior (e.g. pheromone-controlled anemotaxis in moths).

\section{Introduction}

Despite their miniaturized brain, insects are not simple reflex automata; instead, they exhibit a rich behavioral repertoire. Drosophila melanogaster, for example, a tiny insect weighting half of a milligram, has only 100,000 neurons -a million times less than the human brain- and this reduced number of neurons does not prevent the fly from sensory processing and flight maneuvers that are unmatched with current technology $(1,2)$. Understanding how miniaturized insect brains control sensory processing and flight behavior could serve as a source of inspiration for future developments in robotics, e.g., micro-aerial vehicles mimicking flapping flight at the insect scale $(3,4)$ or olfactory robots inspired by odor tracking in moths $(5)$.

For decades, researchers have developed laboratory experimental setups to study and understand the flight behavior of insects. There are two approaches: one consists of maintaining

the insect in position so that flight kinematics can be analyzed in great detail with high-speed cameras, and the other considers more natural conditions, that is, free flight.

Restraining the animal movements can be done in two ways: by taking advantage of the specificity of particular insects, e.g., the ability for hawkmoths to hover in front of artificial flowers while feeding on nectar $(6,7)$ (Fig. 1A), or by physically constraining the insect with a tether, e.g. (8) (Fig. 1C). Both techniques have critical limitations. The former is restricted to studying a steady condition, that is, hovering flight, whereas a rigid tether affects flight dynamics, as it does not allow roll or pitch motions.

In free flight approaches, a few studies attempted to record muscle activities with miniature 
electrophysiology devices carried by the insect during flight (9). Yet, they have been of limited success because the carried load affects flight performance. This approach is thus restricted to the largest insects (e.g. hawkmoths with body mass from 1 to $3 \mathrm{~g}$ ) that have the capability to carry loads up to $10 \%$ of their weight. Wind tunnels (ca. 1 to $2 \mathrm{~m}$ long and $30 \mathrm{~cm}$ wide) equipped with motion capture are common tools to record the trajectories of insects in free flight. They have been used to characterize the attractiveness of insects in turbulent odor plumes such as the anemotactic response of male moths to sexual pheromone; see Reviews $(10,11)$. Flight paths are typically zigzags along the wind axis with decreasing amplitude as the insect approaches the source. Yet, these are only average or indirect observations because wind tunnels do not allow the precise delivery of stimuli in space and time, and the use of external cameras positioned far away from the insect prevents any analysis of flight behavior. Consequently, synchronous measurement of the airborne cues actually encountered by the animal at any point of its displacement and of the animal orientation towards the source is difficult to achieve. Recently, Stower et al. (12) used virtual reality to manipulate the optomotor response of the insect so that the flight is kept within a limited volume and can be tracked with motion capture (Fig. 1B).

Thus, the vast majority of experimental research on insect flight has been limited to the study of hovering flights (Fig. 1A), tethered flights (Fig. 1C), or restricted flights in confined environments (Fig. 1B). Here, we report a cable-driven robot, named lab-on-cables, which endeavors to overcome these limitations. In our approach, the lab equipment is mounted on cables so that it can move along with the animal (Fig. 1D). Cable robots belong to a special class of parallel robots in which rigid links are replaced by flexible cables (13). They offer several advantages, such as the possibility of moving objects with high precision in a large workspace. Famous applications are the SpiderCam or SkyCam (14), a suspended camera moving over stadiums and the RoboCrane (15), a robotic crane used in construction sites. Cable robots have 
also been used as motion simulators for humans (16) and motion generators in wind tunnels (17). The task of moving lab rigs along with a flying insect is challenging because the insect trajectory is not known in advance and the flight speed can be high (several meters per second) (18). In this Article, we describe the design of the lab-on-cables that fulfills this task and its experimental validation.

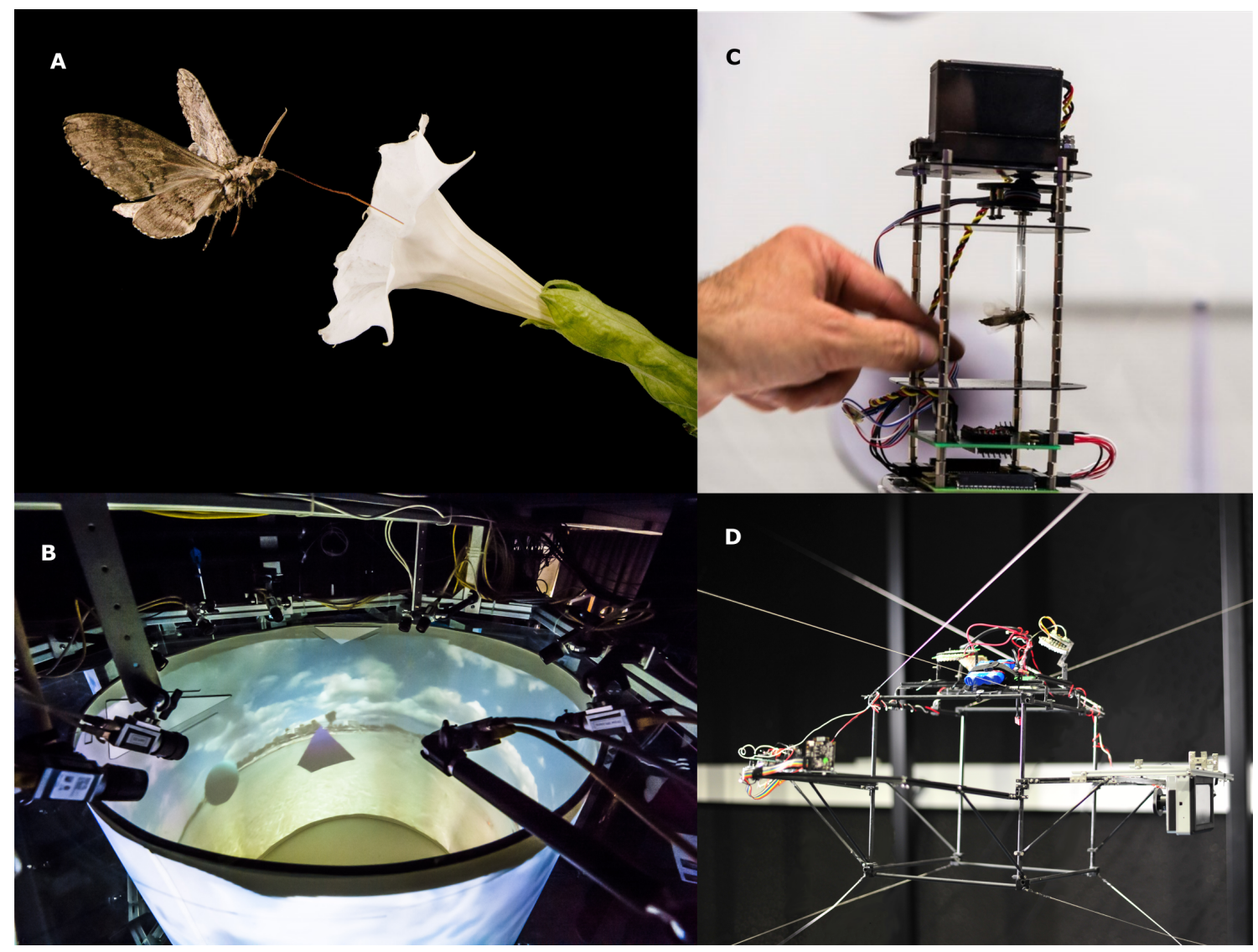

Figure 1: Experimental setups for studies with flying insects. (A) Hovering flight of a Manduca sexta (100-mm wingspan) in front of an artificial flower $(6,7)$. Photo credit: Kiley Riffell photography. (B) Virtual reality flight arena (Drosophila melanogaster) (12). Photo credit: IMP/IMBA Graphics Department, https://strawlab.org/freemovr. (C) Tethered moth (Agrotis ipsilon) from our lab. Photo credit: Patrice Latron photography. (D) Lab-on-cables (A. ipsilon moths) from our lab. All photos used with permission. 


\section{Results}

\section{Lab-on-cables implementation}

We built the lab-on-cables, a 6-degrees of freedom (DOF) cable robot that tracks flying insects in a ( $6 \mathrm{~m}$ long by $4 \mathrm{~m}$ wide by $3 \mathrm{~m}$ high) workspace (Fig. $2 \mathrm{~A}$ and Movie 1). Here, we sketch the main ideas of the design and refer the reader to Materials and Methods for the details. The end effector, called flying frame, is an open cube (edge length of $30 \mathrm{~cm}$ ) in which the insect can fly freely. To follow the insect trajectory, we mounted the flying frame on cables operated by motorized winches (Fig. 2, B and C). An optical system, which computed online the threedimensional (3D) position $\boldsymbol{X}_{\boldsymbol{T}}$ of the insect using infrared (IR) illumination and calibrated cameras, was integrated onto the flying frame. A control strategy that consists of chasing the current target position is risky and can lead off the track because insects may fly at a relatively high speed. Instead, the control scheme takes into account the direction of motion of the insect to anticipate what its future location would be and points towards it (Fig. 2D). This strategy can be seen as a type of deviated pursuit used in missile guidance (19). One major difference, however, is that a missile aiming at target interception flies at its maximum speed, whereas the lab-on-cables tracking an insect adjusts its speed continuously. The tracking speed can be described as follows

$$
\boldsymbol{V}=\boldsymbol{K}_{p}\left(\boldsymbol{X}_{T}-\boldsymbol{X}\right)+\boldsymbol{K}_{d} \dot{\boldsymbol{X}}_{\boldsymbol{T}}
$$

where $\boldsymbol{X}$ is the position of the robot (center of flying frame), $\dot{\boldsymbol{X}}_{T}$ is the insect velocity (time derivatives are indicated with a dot throughout the paper) and $\boldsymbol{K}_{p}$ and $\boldsymbol{K}_{d}$ are proportional and derivative gains. 

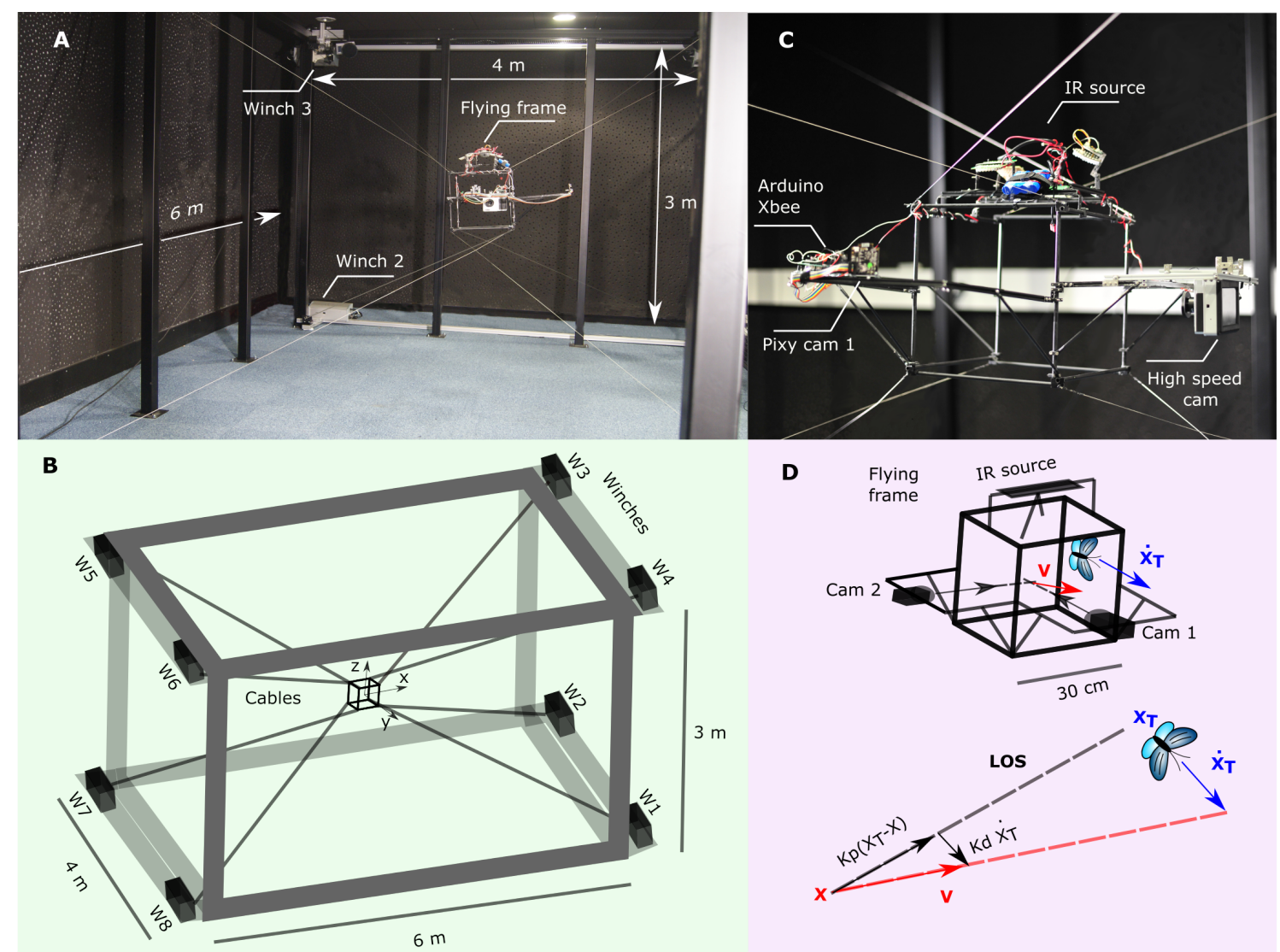

Figure 2: Lab-on-cables setup. (A) Photo of the cable robot (6 $\mathrm{m}$ by $4 \mathrm{~m}$ by $3 \mathrm{~m})$ and (B) Schematic view of the setup. (C) Photo of the flying frame $(30 \mathrm{~cm}$ by $30 \mathrm{~cm}$ by $30 \mathrm{~cm}$ ). The flying frame supports the lab equipment, i.e., an IR source and a pair of calibrated cameras (Pixy cam 1 and 2) for online insect location. The flying frame moves automatically to keep the insect within the detection range of the cameras. (D) Robot control as deviated pursuit. Robot and insect locations are $\boldsymbol{X}$ and $\boldsymbol{X}_{T}$, respectively. The insect speed is denoted $\dot{\boldsymbol{X}}_{\boldsymbol{T}}$. The tracking speed $\boldsymbol{V}$ is the sum of a pure pursuit term pointing along the line of sight (LOS) toward the target current location and a corrective term, taking into account the direction of motion of the target. This is like anticipating where the target will be to point ahead of the target and cover less distance. 


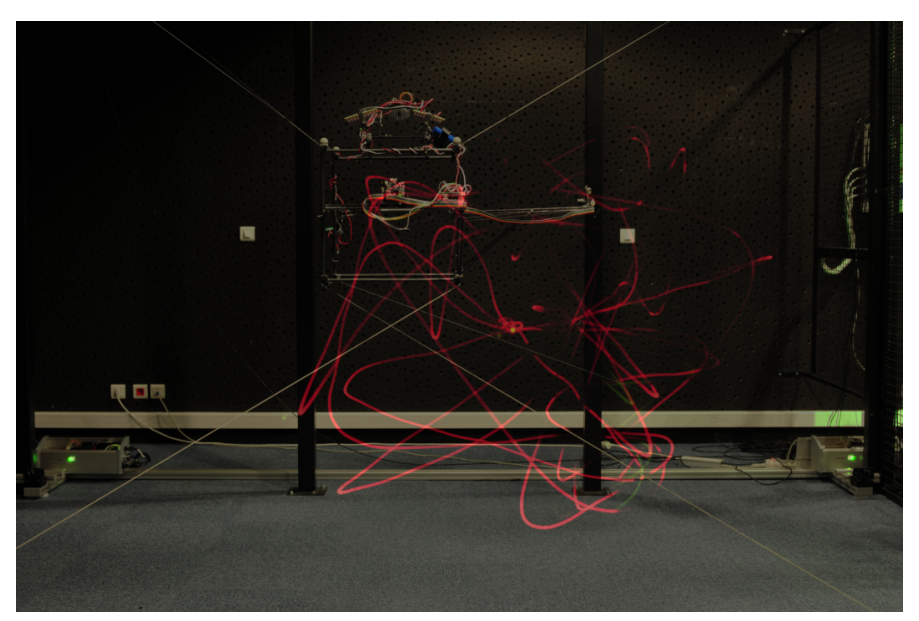

Movie 1. Lab-on-cables at $1 \mathrm{~m} / \mathrm{s}$ superimposed with long-exposure photo of robot movement.

\section{Experimental validation of control design}

We first performed robotic experiments with insect trajectories that had been previously recorded in wind tunnels $(20,21)$. The trajectories corresponded to various insects flying in attractive odor plumes: fruit flies (Drosophila melanogaster, $n=169$ insects) with ethanol plume (20), mosquitoes (Aedes aegypti, $n=65$ insects) with $\mathrm{CO}_{2}$ plume (20) and moths (Agrotis ipsilon, $n=38$ insects) with pheromone plume (21). The objective in replaying prerecorded trajectories was twofold: to conduct pretests with different insects and to bypass the embedded detection system to validate the control design of the robot.

Figure 3 (A to C, left) shows three examples of trajectories tracked by the robot (control gains $K_{p}=8.4$ and $K_{d}=1$ ). The robot was programmed to reach, every $10 \mathrm{~ms}$, a new target position that was read sequentially from the data file of prerecorded trajectory. To test the effectiveness of the robot, we computed the tracking error for all points in all trajectories as the Euclidean distance between the target (flying insect) and the end effector (center of flying frame). Overall, the tracking error increased with the speed of the insect, so that small (or large) errors are made at low (respectively high) speed (Fig. 3, A to C, right). The connected points 
in Fig. 3 (A to $\mathrm{C}$, right) indicate the evolution of the tracking error along the trajectories. We noted, in some trajectories, that large residual errors experienced at high speed persisted from one operating cycle to the next (i.e., $10 \mathrm{~ms}$ ) when the insect slowed down. This observation suggests some limitations for the robot in terms of speed and acceleration. To assess these limits, we used speed-step control inputs and analyzed the transient response of the robot. We estimated the robot limitations to $3.6 \mathrm{~m} / \mathrm{s}$ and $17 \mathrm{~m} / \mathrm{s}^{2}$.

For all species, the error distribution is well described by a gamma distribution with fitted shape and scale parameters. The cumulative distributions plotted in Fig. 3D for the various insects indicate that, in more than $90 \%$ of the cases, the tracking error was less than $1 \mathrm{~cm}$, which is small compared with the length of the flying frame $(30 \mathrm{~cm})$. The experimental tracking errors could be reproduced in simulation by incorporating the speed and acceleration limits into a simple model of the robot (compare Fig. 3, E and C, right). The simulated model merely consisted in integrating Eq. 1 using Euler method. The fact that Eq. 1 can account for the behavior of the robot validates the control design. Among the three species, the trajectories of A. ipsilon moths are the most difficult to follow; see intermittent large errors $(\mathrm{ca} .10 \mathrm{~cm})$ due to high flight speeds (ca. $5 \mathrm{~m} / \mathrm{s}$ ) in Figure 3C (right). In the next section, we use the lab-on-cables to track free-flying A. ipsilon moths.

\section{Tracking free-flying A. ipsilon moths and analysis of flight kinematics}

A prerequisite to tracking flying insects is the takeoff procedure depicted in Fig. 4A (see also movie S1). The insect is gently placed onto a takeoff platform $\left(2 \mathrm{~cm}^{2}\right)$ positioned near the center of the flying frame, and the robot starts tracking immediately. If spontaneous takeoff does not occur within $5 \mathrm{~min}$, insect flight is initiated by thermal stimulation (Peltier element on the takeoff platform). After takeoff, an electromagnet allows the fall of the platform in order not to hamper the tracking of the insect by the robot. We did not notice any difference between 
A

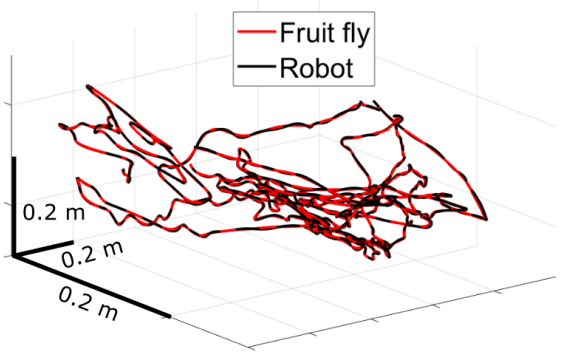

B

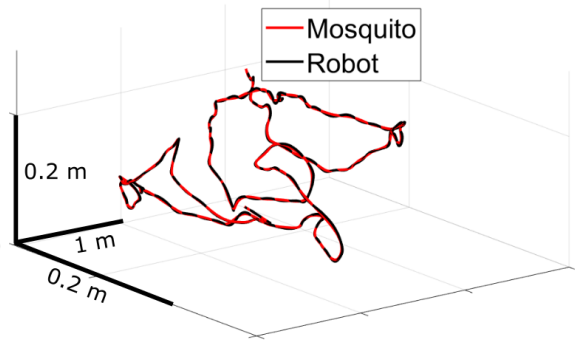

C

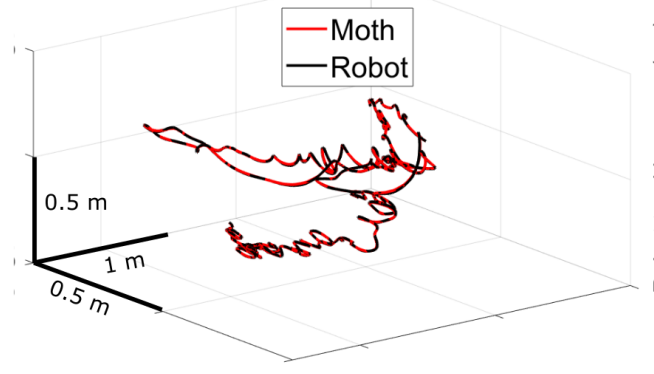

D Tracking error distribution

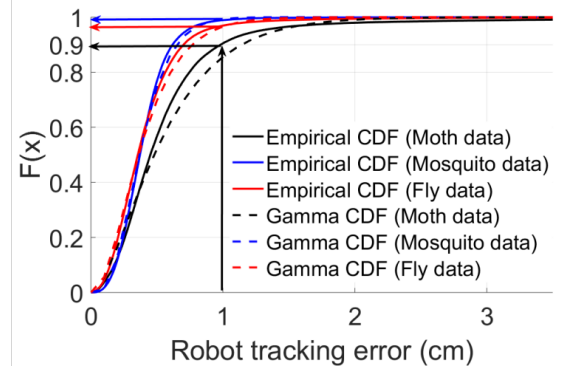

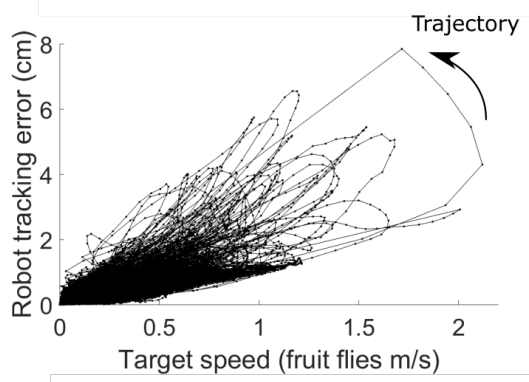

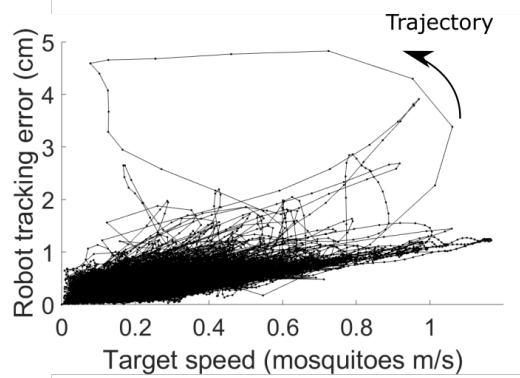

Target speed (mosquitoes $\mathrm{m} / \mathrm{s}$ )

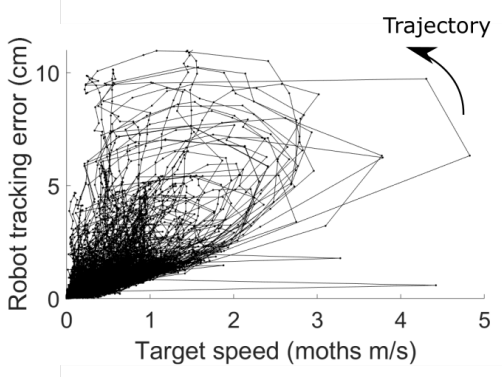

E Simulations

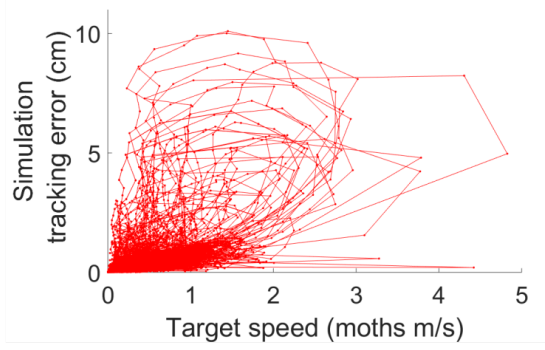

Figure 3: Tracking prerecorded insect trajectories. (A to C) Left: Examples of insect trajectories (red curves) versus robot trajectories (black curves). Right: Tracking errors of the robot (in centimeters) versus flight speed of the insect (in meters per second). (D) Cumulative distributions of the tracking error for experimental data (plain curves) and theoretical gamma distribution (dashed curves). (E) Tracking errors of the simulated model (Euler integration of Eq. 1) versus flight speed of the insect (moth data). 
spontaneous takeoffs and those provoked by thermal stimulation. In either case, A. ipsilon moths jumped from the platform by using both their wings and legs to power the takeoff. This flight initiation is similar to one of the jumping strategies identified for moths in (22).

We performed tracking experiments with $n=32$ moths. During flight, the insect is located online from the embedded optical system, and therefore, in contrast to previous experiments, the target position is noisy. To improve stability in the presence of noise, we reduced the proportional and derivative gains to $K_{p}=3$ and $K_{d}=0.9$. The flight trajectories ranged from 0.5 to $3 \mathrm{~m}$ (Fig. 4B). The cumulative distribution in Fig. 4B indicates that the insect speed during flight is lower than $3 \mathrm{~m} / \mathrm{s}$ in $99 \%$ of the cases.

For $n=6$ moths (shown as colored points in Fig. 4B), we recorded images at 400 frames per second (fps) (i.e., 10 times the wingbeat frequency of A. ipsilon moths) with a high-speed camera mounted on the flying frame (Fig. 5A, left). To extract the body kinematics from the video sequence, we modeled A. ipsilon moth in 3D using Blender, a computer graphics software (Fig. 5A, right). The model was rigged to be fully articulated; i.e., the position and rotation of body and wings could be set freely under the constraint of symmetry between the left and right wings. The position and rotation of body and wings were optimized semi-automatically, so as to match those of the real insect in each image of the recorded video sequences (see Materials and Methods). From the fitted model, we extracted the body kinematics represented by the body angle $\alpha$ and the stroke plane angle $\beta$ with respect to the horizontal plane, as depicted in Fig. 5A (right). The stroke plane was identified by least square fitting from the positions of the wing base and tip during an entire wing beat (i.e., approximately 10 consecutive 3D points).

We analyzed the kinematics for six moths representing a total of 154 wingbeats (Movie 2). The wingbeat frequency as well as body and stroke plane angles change with the flight speed. As flight speed increases from 0 to $3 \mathrm{~m} / \mathrm{s}$, the wing beat frequency increased from 39 to $47 \mathrm{~Hz}$ $[P<0.05$, one-way analysis of variance (ANOVA); Fig. $5 \mathrm{~B}]$ while the insect body tends to 
be be more horizontal, with $\alpha$ decreasing from $59^{\circ}$ to $15^{\circ}[P<0.001$, one-way ANOVA; Fig. 5C]. The opposite is observed in Fig. 5D for the stroke plane angle, with $\beta$ increasing from $25^{\circ}$ to $57^{\circ}(P<0.001$, one-way ANOVA). Body and stroke plane angles are not independent. We note in Fig. 5E that $\alpha=-\beta+82^{\circ}$ (Pearson correlation $R^{2}=0.74$ ), so that the angle $\alpha+\beta$ between the stroke plane and the longitudinal axis of the body is approximately constant. These results are consistent with those obtained in (6), albeit in different situations: hovering moths in the presence of a steady air flow versus moths flying freely with variable acceleration and repeated changes of direction in our study.
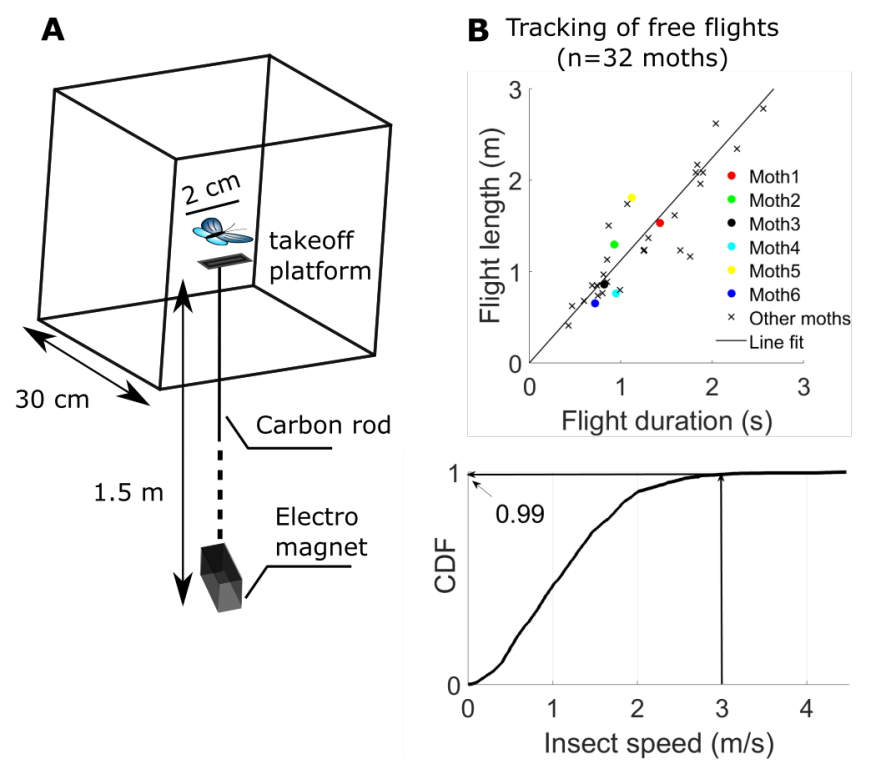

Figure 4: Tracking A. ipsilon moths. (A) Takeoff procedure. (B) Tracking of free flights ( $n=32$ moths). Top: Flight length versus flight duration fitted by linear regression (Peason correlation $R^{2}=0.79$ ). The colored points are for the six moths used in the analysis of the flight kinematics. Bottom: Cumulative density function (CDF) of the insect flight speed during tracking. 
A Analysis of flight kinematics ( $n=6$ moths)
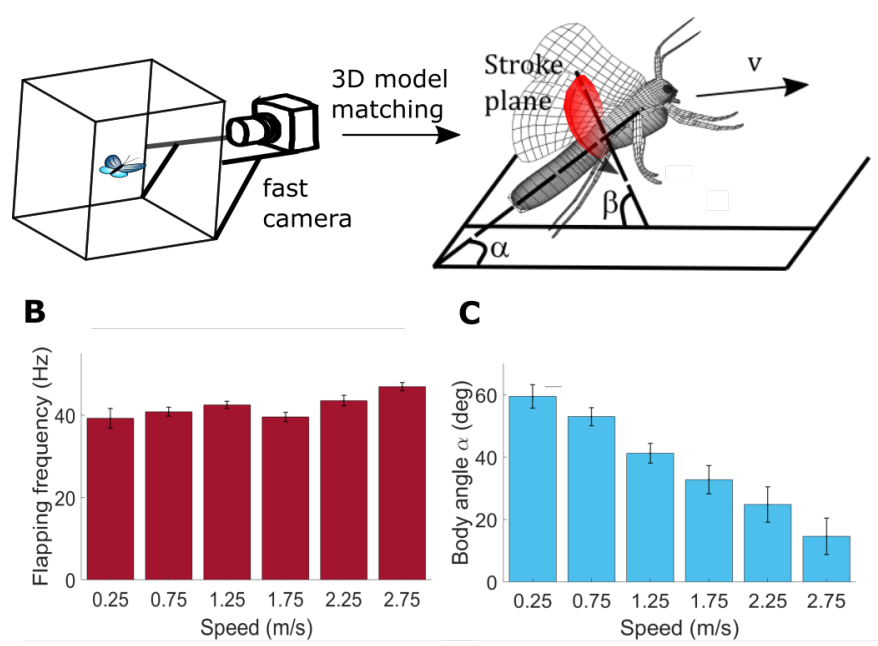

C
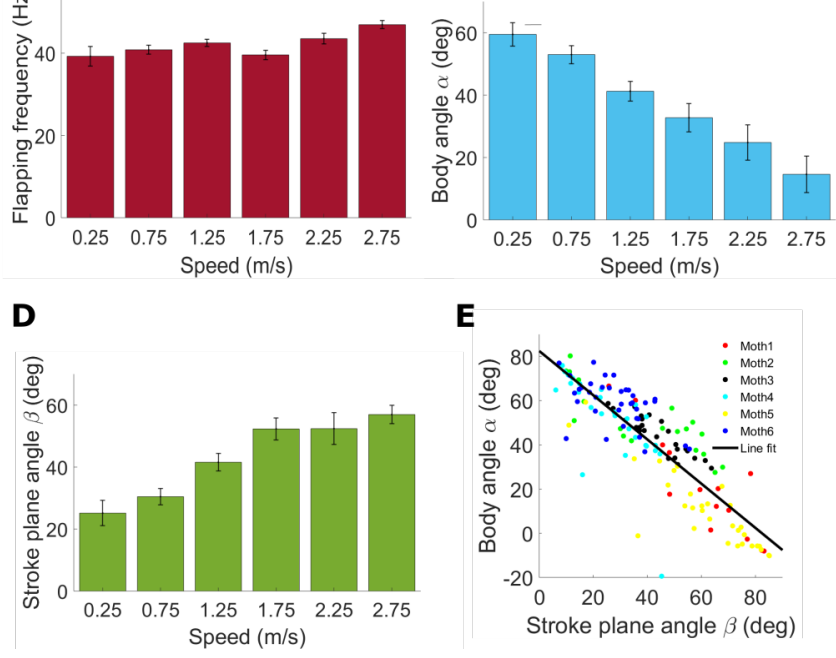

$\mathbf{E}$

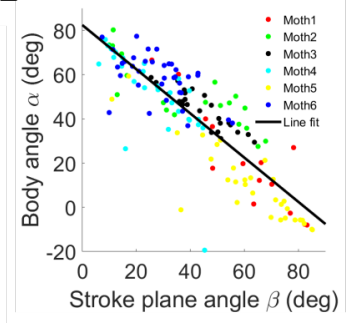

Figure 5: Analysis of flight kinematics. (A) A high-speed camera is mounted on the flying frame, and the pose of the 3D model of the moth is optimized semi-automatically to match the one of the real insect in the recorded videos. The inclination of the body is represented by the body angle $\alpha$, i.e., the angle between the longitudinal axis of the body and the horizontal plane. The angle $\beta$ is between the horizontal and the stroke plane defined by the positions of wing base and tip during an entire wing beat. (B) Wingbeat frequency versus flight speed. (C) Body angle versus flight speed. (D) Stroke plane angle versus flight speed. In (B) to (D), the mean values \pm SE represent averages within speed intervals (bin size, $0.5 \mathrm{~m} / \mathrm{s}$ ). (E) Body angle versus stroke plane angle. 


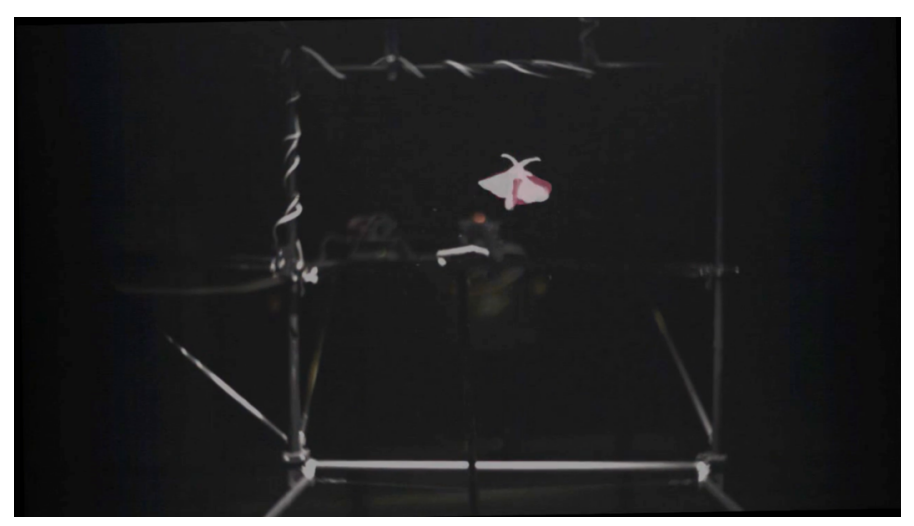

Movie 2. Tracking A. ipsilon moths with extraction of flight kinematics by 3D model-based matching (real moth in white and 3D model in pink).

\section{Discussion}

We validated the lab-on-cables with $A$. ipsilon moths flying freely up to $3 \mathrm{~m} / \mathrm{s}$ and measured the moth kinematics with an on-board high-speed camera. The data analysis indicates that, when flight speed increases, A. ipsilon moths pitch their body down while the stroke plane becomes more vertical, as body and stroke plane angles vary in accordance. This result is consistent with a helicopter model of insect flight $(6,23,24)$, whereby flight speed is controlled by body pitch via changes in the stroke plane angle.

Measurements of insect flight with high-speed cameras are usually hampered by the tradeoff between spatial resolution and field of view, which requires the insect to stay in the vicinity of the camera system. The insect is thus traditionally kept within the detection range (typically a few tens of centimeters) by imposing some sort of movement restriction, whether by means of a tether or by confining the flight space. With the lab-on-cables, the insect evolves freely in an open space, albeit with a detection range that is constrained by the size of the workspace. In its present form, the lab-on-cables was located indoor in a relatively small room, which limits the operational workspace; however, because it relies on a lightweight cable structure and mechanically simple winches, one can envisage rescaling the design to perform experiments at 
a larger scale and even in the wild.

When studying flying insects, we also need to bear in mind that experimental setups may lead to unnatural flight behavior. This is known for tethered insects which tend to exaggerate their movements because they do not support their own body mass, and for insects flying in confined environments which modulate their speed according to the distance from the wall (18). The lab-on-cables is not likely to alter the flight kinematics, because our results are consistent with previous studies in free flight. Yet, it could be argued that the flying frame might still be considered a threat by the insect. However, it is worth noting that the flying frame moves with the insect, so the situation is totally different from a looming stimulus produced by a rapidly approaching predator. If the flying frame had been perceived as a threat, evasive maneuvers would have been observed in the flight of moths, and our experiments do not support this claim. The typical escape behavior in moths, which consists in cessation of flight and dropping to the ground, was not observed in any of the 32 moths tested.

We are not aware of any apparatus or concept comparable to the lab-on-cables, which has thereby the potential to become an important tool in the study of flying insects. The lab-oncables uses the advantages of cable robots to track flying insects, namely, very fast dynamics with little air disturbance and the possibility to be deployed over a large workspace, thanks to the lightweight cable structure. Moreover, taking other equipment on-board would allow for stimulus delivery during flight so that one could then study sensory-driven behaviors in an unprecedented manner. A typical example would be to study pheromone-controlled anemotaxis in moths with a precise control of the olfactory cues during their flight. Despite the advantages of the lab-on-cables, there is still room for performance improvement in this technology. For example, the use of cameras with increased spatial and temporal resolution may prove beneficial to limit noise in the estimation of the target position. Similarly, the use of more powerful motors can push forward the maximum attainable limits in speed and acceleration (currently estimated 
at $3.6 \mathrm{~m} / \mathrm{s}$ and $17 \mathrm{~m} / \mathrm{s}^{2}$, respectively). Such technological adaptations are feasible, because ultrafast cable robots have been reported [e.g., the Falcon robot with velocities and accelerations up to $13 \mathrm{~m} / \mathrm{s}$ and $430 \mathrm{~m} / \mathrm{s}^{2}$, respectively (25)], and thus would be useful as future additions.

\section{Materials and Methods}

\section{Hardware design}

We built the cable robot partly from hardware provided by Haption (26). The base frame is a parallelepiped of dimensions $6 \mathrm{~m}$ by $4 \mathrm{~m}$ by $3 \mathrm{~m}$ (Fig. 2, A and B). The robot with eight cables for $6 \mathrm{DOF}$ is overconstrained. The cables are driven by motorized winches (Maxon DC motor RE65 with COMBIPERM P1-03 brake) located at the corners of the base frame. The cables have anchor points at the vertices of the end effector (flying frame) (Fig. 2C). As in previous designs [e.g. (27)], the cables are crossed in a way that increases the stiffness of the system. Because no cable goes through the flying frame, it represents a safe environment for the insect to fly in. The flying frame supports laboratory rigs, that is, a stereo-vision system for online insect tracking.

The IR filter was removed from all cameras and scene illumination was provided by an IR source. The IR source did not disturb the insect because its intensity spectrum (Fig. 6, inset), measured with a spectrometer (Ocean Optics STS-NIR 650-1100 nm), is well beyond the detection range of insect photoreceptors (28). For online insect tracking, we embedded two calibrated Pixy cameras (CMUcam5) that captured images and extracted the 2D pixel coordinates (effective resolution of 320 x 200 pixels) of the insect at $50 \mathrm{fps}$. The $2 \mathrm{D}$ pixel coordinates of the two cameras were transmitted wirelessly to the control computer using Arduino UNO and Xbee. The 3D triangulation was performed based on a least-square fit of the intersection between the two lines derived from the $2 \mathrm{D}$ pixel coordinates and the $3 \mathrm{D}$ camera centers. The triangulation result was filtered with a constant velocity Kalman filter (29) to provide the 3D 
position $\left(x_{T}, y_{T}, z_{T}\right)$ of the target (insect).

\section{Control design}

The control design of the robot follows classical methods for cable robots, as described in (13). The control scheme is outlined in Fig. 6 and consists of four steps.

- First, the combination of position and orientation of the flying frame -referred to as the pose, $\mathbf{X}=(x, y, z, \alpha, \beta, \gamma)^{T}$, with $(\alpha, \beta, \gamma)$ representing yaw, pitch and roll angles, respectivelyis estimated from the cable lengths by solving the forward kinematic problem. This approach is preferable to a motion capture system because it does not rely on markers, i.e., retroreflective materials placed on the flying frame. Thus, it covers a larger workspace with increased robustness (no failure due to occluded markers as in optical motion capture systems). To quantify the positioning error, we estimated the position of the flying frame from the cable lengths at each time step on random walk trajectories and compared it to ground truth data provided by a motion capture system (Qualisys Mocap 6 cameras Oqus 700+). The positioning error was 1.5 $\pm 0.6 \mathrm{~cm}$, which is small compared with the length of the flying frame (i.e., $30 \mathrm{~cm}$ ).

- Second, the tracking speed is determined by a controller acting on the tracking error, as defined by the distance between the target (flying insect) and the end effector (center of flying frame). Here, we consider that the target pose is $\mathbf{X}_{\mathbf{T}}=\left(x_{T}, y_{T}, z_{T}, 0,0,0\right)^{T}$, because the aim of the controller is to track the insect position while maintaining a zero orientation (flying frame aligned to base frame). The tracking speed is given by Eq. 1 with diagonal matrices $\boldsymbol{K}_{\boldsymbol{p}}=\operatorname{diag}\left(K_{p}, K_{p}, K_{p}, 1,1,1\right)$ and $\boldsymbol{K}_{\boldsymbol{d}}=\operatorname{diag}\left(K_{d}, K_{d}, K_{d}, 0,0,0\right)$. The proportional and derivative gains $K_{p}$ and $K_{d}$ have been set by using Ziegler-Nichols initial estimation (30) followed by manual fine tuning.

- Third, the inverse kinematic model allows us to convert the tracking speed into a (winding/unwinding) speed vector for the winches which can be written as $\dot{\ell}=\boldsymbol{J} \boldsymbol{V}$ with $\boldsymbol{J}$ being 
the Jacobian matrix. The Jacobian matrix can be seen as a local linearization of the system at the current pose $\mathbf{X}$. Its expression is given in supplementary text.

- Fourth, the winding/unwinding speed vector is converted into a motor speed command with constraints on the cable tension. It is necessary to ensure a sufficient tension in the cables to prevent sagging (as forward and inverse kinematics consider taut cables) while avoiding, at the same time, an excessive tension that can lead to cable breaking. This optimization problem is framed as a Quadratic Programming problem (see supplementary text). The motor speed command is sent to the motorized winches every $10 \mathrm{~ms}$.

The methods used for solving the forward and inverse kinematics problems in the lab-oncables and determining the suitable tension in the cables are derived from a textbook (13). They are detailed in supplementary text.

\section{Extraction of flight kinematic variables by 3D-model-based matching}

For $n=6$ A. ipsilon flights, we recorded images at $400 \mathrm{fps}$ with an embedded high-speed camera (FPS 4000 with $22 \mathrm{~mm}$ lens). To extract flight kinematics from the video sequence, we modeled A. ipsilon moths in 3D using Blender (Fig. 5A). The model was rigged to be fully articulated; i.e., the position and rotation of body and wings could be set freely. We also modeled the flying frame and used the same parameters (position and focal length) for the Blender camera as those of the high-speed camera. The video sequence was also preprocessed by increasing brightness and contrast. All these adjustments ensured a relatively realistic render which accurately matched the real video sequence. The next step was to achieve semi-automatic shape matching between the 3D model and the real moth in the video sequence. To process the video sequence, we applied the 3D position of the insect provided by the Pixy cameras to the model every 10 frames. These positions were then manually corrected if needed, and the remaining frames were interpolated (Bézier). The rotation of the body and wings was set 


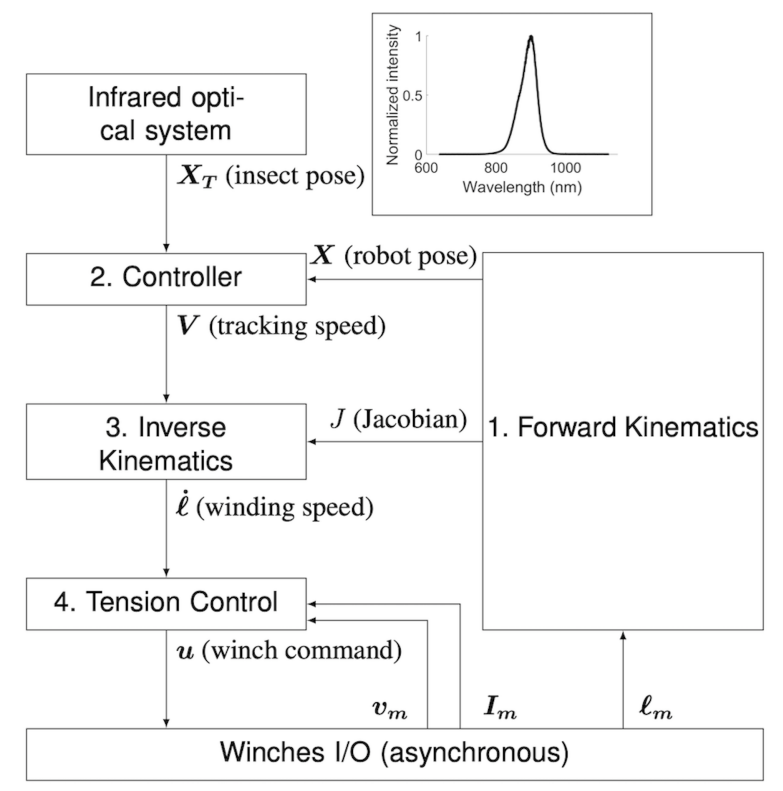

Figure 6: Control scheme. After insect detection by the IR optical system (normalized intensity spectrum provided in the inset), the control scheme of the robot consists in four steps: (1) estimation of the robot pose by using forward kinematics, (2) computation of the tracking speed to minimize the tracking error, (3) transformation into a winding speed vector by using the Jacobian and inverse kinematics, and (4) conversion to motor command with constraints on the cable tensions. The vectors $\boldsymbol{I}_{\boldsymbol{m}}=\left(I_{1 m}, \cdots, I_{8 m}\right)^{T}, \boldsymbol{\ell}_{\boldsymbol{m}}=\left(\ell_{1 m}, \cdots, \ell_{8 m}\right)^{T}$ and $\boldsymbol{v}_{\boldsymbol{m}}=$ $\left(v_{1 m}, \cdots, v_{8 m}\right)^{T}$ are measurements of the motor currents, of the winding/unwinding speeds, and of the cable lengths, respectively. 
manually until the superimposed model matched the insect in the frame. We considered that the wings always remain symmetrical. The flying frame was also animated to reproduce the real robot movement. Once matching was achieved, the whole video sequence could be rendered and model parameters could be extracted (positions of the head, tail, center of mass, and base and tip of both wings); see Movie 2.

\section{Supplementary materials}

Text S1. Details on the control of the robot.

Fig. S1. Geometry of the lab-on-cables.

Movie S1. Insect take-off

\section{References}

1. D. Floreano, J.-C. Zufferey, M. V. Srinivasan, C. Ellington, Flying Insects and Robots, Springer, (2009).

2. D. Floreano, R.J. Wood, Science, technology and the future of small autonomous drones, Nature 521, 460-466 (2015).

3. D. Lentink, Flying like a fly, Nature 498, 306 (2013).

4. K. Y. Ma, P. Chirarattananon, S. B. Fuller, R. J. Wood, Controlled flight of a biologically inspired, insect-scale robot, Science 340, 604 (2013).

5. D. Martinez, On the right scent, Nature 445, 371 (2007). 
6. A. P. Willmott, C. P. Ellington, The mechanics of flight in the hawkmoth Manduca sexta. I. kinematics of hovering and forward flight., Journal of Experimental Biology 200, 2705 (1997).

7. T. L. Hedrick, T. Daniel, Flight control in the hawkmoth Manduca sexta: the inverse problem of hovering, Journal of Experimental Biology 209, 3114 (2006).

8. B. J. Duistermars, D. M. Chow, M. Condro, M. A. Frye, The spatial, temporal and contrast properties of expansion and rotation flight optomotor responses in Drosophila, Journal of Experimental Biology 210, 3218 (2007).

9. N. Ando, I. Shimoyama, R. Kanzaki, A dual-channel fm transmitter for acquisition of flight muscle activities from the freely flying hawkmoth, Agrius convolvuli, Journal of Neuroscience Methods 115, 181 (2002).

10. R. T. Cardé, M. A. Willis, Navigational strategies used by insects to find distant, wind-borne sources of odor, Journal of Chemical Ecology 34, 854 (2008).

11. N. J. Vickers, Winging It: Moth Flight Behavior and Responses of Olfactory Neurons Are Shaped by Pheromone Plume Dynamics, Chemical Senses 31, 155 (2005).

12. J. R. Stowers, et al., Virtual reality for freely moving animals, Nature Methods 14, 995 (2017).

13. A. Pott, Cable-driven parallel robots: theory and application, vol. 120 (Springer, 2018).

14. L. L. Cone, Skycam, an aerial robotic camera system, Byte Magazine 10, 122 (1985).

15. N. G. Dagalakis, J. S. Albus, B.-L. Wang, J. Unger, J. D. Lee, Stiffness study of a parallel link robot crane for shipbuilding applications, ASME Journal of Mechanical Design 111, 183 (1989). 
16. P. Miermeister, et al., 2016 IEEE/RSJ International Conference on Intelligent Robots and Systems (IROS) (IEEE, 2016), pp. 3024-3029.

17. P. Lafourcade, M. Llibre, C. Reboulet, Proceedings of the Workshop on Fundamental Issues and Future Research Directions for Parallel Mechanisms and Manipulators (Quebec City, Canada, 2002), pp. 187-194.

18. R. Stevenson, K. Corbo, L. Baca, Q. Le, Cage size and flight speed of the tobacco hawkmoth Manduca sexta, Journal of Experimental Biology 198, 1665 (1995).

19. G. M. Siouris, Missile guidance and control systems (Springer Science \& Business Media, 2004).

20. R. Pang, F. van Breugel, M. Dickinson, J. A. Riffell, A. Fairhall, History dependence in insect flight decisions during odor tracking, PLOS Computational Biology 14, 1 (2018).

21. F. Dupuy, et al., A background of a volatile plant compound alters neural and behavioral responses to the sex pheromone blend in a moth, Frontiers in Physiology 8, 79 (2017).

22. M. Burrows, M. Dorosenko, Jumping mechanisms and strategies in moths (Lepidoptera), Journal of Experimental Biology 218, 1655 (2015).

23. C. David, The relationship between body angle and flight speed in free-flying Drosophila, Physiological Entomology 3, 191 (1978).

24. V. Medici, S. Fry, Embodied linearity of speed control in Drosophila melanogaster, Journal of The Royal Society Interface 9, 3260 (2012).

25. S. Kawamura, W. Choe, S. Tanaka, H. Kino, Development of an ultrahigh speed robot falcon using parallel wire drive systems, Journal of the Robotics Society of Japan 15, 82 (1997). 
26. J. Perret, L. Dominjon, Joint Virtual Reality Conference, Springer Tracts in Advanced Robotics (2009).

27. L. Gagliardini, S. Caro, M. Gouttefarde, 2015 IEEE International Conference on Automation Science and Engineering (CASE) (IEEE, 2015), pp. 99-105.

28. D. Osorio, M. Vorobyev, Photoreceptor sectral sensitivities in terrestrial animals: adaptations for luminance and colour vision, Proceedings of the Royal Society B: Biological Sciences 272, 1745 (2005).

29. A. D. Straw, K. Branson, T. R. Neumann, M. H. Dickinson, Multi-camera real-time threedimensional tracking of multiple flying animals, Journal of The Royal Society Interface $\mathbf{8}$, 395 (2011).

30. J. G. Ziegler, N. B. Nichols, et al., Optimum settings for automatic controllers, Transaction of American Society of Mechanical Engineers 64 (1942).

Acknowledgements: We thank Michel Renou for providing the pre-recorded moth trajectories and Sooraj Sivakumar for help on visual target detection. Funding: This research was supported by the French research program CPER Cyberentreprises (2015-2020), with participation from the Lorraine region and FEDER, and by a PEPS INS2I project from the CNRS. Author contributions: R.P, M.B. and D.M. designed the robot. R.P. and M.B. designed the control. M.J. designed the 3D model of the moth. R.P., P.L. and D.M. conducted the experiments. R.P., M.J. and D.M. analyzed and interpreted the data. R.P, M.J. and D.M. wrote the original draft. R.P., M.J., M.B., P.L. and D.M. revised the manuscript. M.B. and D.M. provided funding and supervised the project. Competing interests: The authors declare that they have no competing interests. Data and materials availability: All data needed to evaluate the conclusions in the article are present in the paper or the Supplementary Materials. 


\title{
Supplementary text for
}

\section{Lab-on-cables: Automatic tracking of free-flying insects}

\author{
Rémi Pannequin ${ }^{1}$, Mélanie Jouaiti ${ }^{2}$, Mohamed Boutayeb ${ }^{1}$, \\ Philippe Lucas ${ }^{3}$, and Dominique Martinez ${ }^{2, *}$ \\ ${ }^{1}$ Université de Lorraine, CRAN, 54000 Nancy, France \\ ${ }^{2}$ Université de Lorraine, CNRS, LORIA, 54000 Nancy, France \\ ${ }^{3}$ Institute of Ecology and Environmental Sciences, INRA, 78000 Versailles, France
}

*To whom correspondence should be addressed; E-mail: Dominique.Martinez@loria.fr

General methods for solving the forward and inverse kinematics problems in cable robots and determining the suitable tension in the cables can be found in the textbook (1). The application of these methods to our particular lab-on-cables design is detailed in this Supplementary text in which the following notations are used: scalar variables are indicated by lowercase letters, e.g., $x$. Time derivatives are indicated with a dot, e.g., $\dot{x}:=\frac{d x}{d t}$. Vectors and matrices are indicated by boldface letters, e.g., $\mathbf{X}$ with $\mathbf{X}^{T}$ denoting its transpose. Euclidean vectors connecting an initial point $X$ with a terminal point $Y$ are denoted by arrows $\overrightarrow{X Y}$.

\section{Geometric model}

The geometry of the lab-on-cables is detailed in Figure S1. Given the two components (mobile end-effector and fixed base-frame), we consider two coordinate systems: 
- A fixed frame of reference, centered at the origin $O_{f}=(0,0,0)^{T}$ of the base frame.

- A mobile frame of reference, centered in the middle of the flying frame at the point $O_{m}$ with coordinates $(x, y, z)^{T}$. The rotation of the flying frame is specified by yaw, pitch and roll angles, i.e. rotations along the $z, y$ and $x$ axes, respectively. The pose of the flying frame is determined by the vector $\mathbf{X}=(x, y, z, \alpha, \beta, \gamma)^{T}$.

Change in the coordinate system, from mobile to fixed, is performed with the translation vector $\overrightarrow{O_{f} O_{m}}=(x, y, z)^{T}$ and the rotation matrix $\mathbf{R}$ given below:

$$
\mathbf{R}=\left(\begin{array}{ccc}
\cos \alpha \cos \beta & \cos \alpha \sin \beta \sin \gamma-\sin \alpha \cos \gamma & \cos \alpha \sin \beta \cos \gamma+\sin \alpha \sin \gamma \\
\sin \alpha \cos \beta & \sin \alpha \sin \gamma+\cos \alpha \cos \gamma & \sin \alpha \sin \beta \cos \gamma-\cos \alpha \sin \gamma \\
-\sin \beta & \cos \beta \sin \gamma & \cos \alpha \cos \gamma
\end{array}\right)
$$

Consequently, the target $T$ (i.e. flying insect) has coordinates $X_{T}=\left(x_{T}, y_{T}, z_{T}\right)^{T}$, expressed in the mobile reference frame, and $X_{T}=\overrightarrow{O_{f} O_{m}}+\mathbf{R} X_{T}$ in the fixed reference frame. In Figure S1, the points $A_{i}, i=1 \cdots 8$, in the base frame correspond to the positions of the pulleys at the entrance of the winches and the points $B_{i}$ are the vertices of the flying frame. The latters are denoted $B_{\text {iref }}$ when the flying frame is aligned to the base frame, that is, when the yaw, pitch and roll angles are zero. The relationship between the two is expressed as

$$
\overrightarrow{O_{m} B_{i}}=\mathbf{R} \overrightarrow{O_{m} B_{i \mathrm{ref}}}
$$

The cables are indexed according to the winches that pull them, i.e. the $i$ th cable connects the point $A_{i}$ of the $i$ th winch to a particular vertex $B_{i}$ of the flying frame. The cables are crossed as in Figure S1. Given the above considerations, the geometry of the lab-on-cables (Fig. 1) is completely determined by:

- the coordinates of the pulley points $A_{i}$ expressed in the fixed frame of reference from the dimensions $H_{1}, H_{2}$ and $H_{3}$ of the base frame (parallelepiped), e.g. $A_{1}=\left(H_{1}, 0,0\right)^{T}$, 
- the coordinates of the anchor points $B_{i}$ expressed in the mobile frame of reference from the edge length $C$ of the flying frame (cube), e.g. $B_{1}=\left(\frac{C}{2}, \frac{C}{2},-\frac{C}{2}\right)^{T}$.

The length of the $i$-th cable is denoted by $\ell_{i}=\left\|\overrightarrow{A_{i} B_{i}}\right\|$. Its winding/unwinding speed is $\dot{\ell}_{i}$ with $\dot{\ell}_{i}<0$ when the cable winds and $\dot{\ell}_{i}>0$ when it unwinds. The unit vector in the direction of the $i$-th cable is denoted by

$$
\overrightarrow{n_{i}}=\frac{\overrightarrow{A_{i} B_{i}}}{\left\|\overrightarrow{A_{i} B_{i}}\right\|}
$$

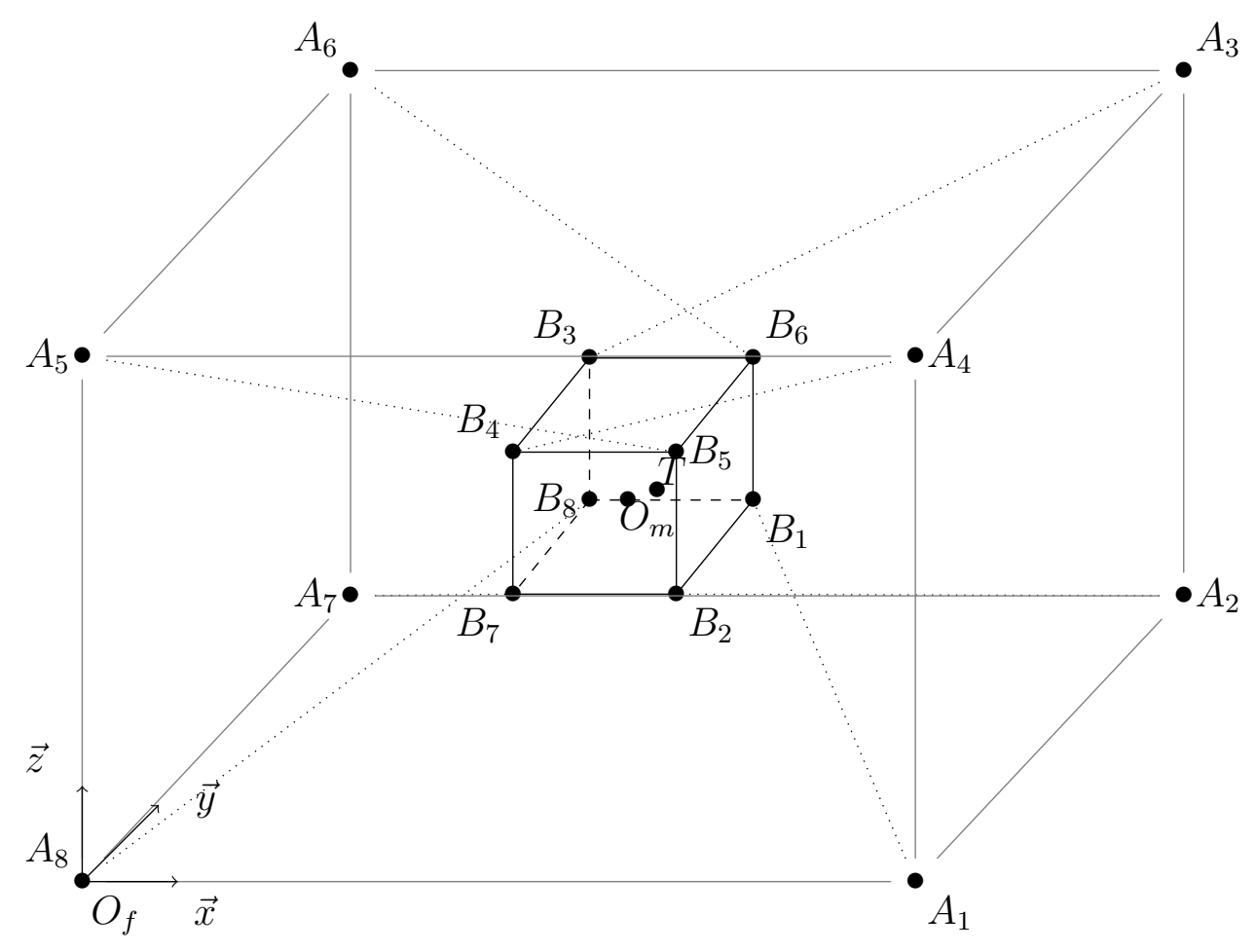

Figure S1: Geometry of the lab-on-cables. The vertices of the flying frame are at the points $B_{i}$, $i=1 \cdots 8$, and the target (i.e. flying insect) at the point $T$. The origin of the fixed and mobile frame of reference is $O_{f}$ and $O_{m}$, respectively. The dashed lines represent the cables changing the pose of the flying frame via motorized winches. The $\mathrm{i}$-th cable connects the points $A_{i}$ corresponding to the position of the pulley at the entrance of the i-th winch to the distal anchor point $B_{i}$. 


\section{Inverse kinematics and Jacobian matrix}

The inverse kinematic problem consists in finding the cable length vector $\ell=\left(\ell_{1} \cdots \ell_{8}\right)^{T}$ for a given pose $\mathbf{X}=(x, y, z, \alpha, \beta, \gamma)^{T}$ of the end-effector, i.e. flying frame. We thus define $f$ as the function providing the cable lengths given the pose; that is, $\ell=f(\mathbf{X})$ where each $i$-th element of $\ell$ is $\ell_{i}=\left\|\overrightarrow{\ell_{i}}\right\|$ with

$$
\overrightarrow{\ell_{i}}=\overrightarrow{O_{f} O_{m}}+\overrightarrow{O_{m} B_{i}}-\overrightarrow{O_{f} A_{i}}
$$

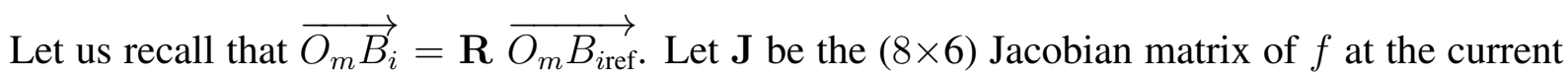
pose $\mathbf{X}$ so that $\dot{\ell}=\mathbf{J} \dot{\mathbf{X}}$. The Jacobian matrix is written as:

$$
\mathbf{J}=\left(\begin{array}{cccccc}
\frac{\partial \ell_{1}}{\partial x} & \frac{\partial \ell_{1}}{\partial y} & \frac{\partial \ell_{1}}{\partial z} & \frac{\partial \ell_{1}}{\partial \alpha} & \frac{\partial \ell_{1}}{\partial \beta} & \frac{\partial \ell_{1}}{\partial \gamma} \\
\cdots & \cdots & \ldots & \cdots & \ldots & \ldots \\
\frac{\partial \ell_{8}}{\partial x} & \frac{\partial \ell_{8}}{\partial y} & \frac{\partial \ell_{8}}{\partial z} & \frac{\partial \ell_{8}}{\partial \alpha} & \frac{\partial \ell_{8}}{\partial \beta} & \frac{\partial \ell_{8}}{\partial \gamma}
\end{array}\right)
$$

To derive the Jacobian matrix, we consider the time derivative of Eq. (1), that is

$$
\overrightarrow{\overrightarrow{\ell_{i}}}=\overrightarrow{O_{f} O_{m}}+\overrightarrow{O_{m} B_{i}}
$$

since $\overrightarrow{O_{f} A_{i}}=0$. Moreover, $\overrightarrow{O_{m} B_{i}}$ is the motion of a fixed point on a rigid body; that is, $\overrightarrow{O_{m} B_{i}}=-\overrightarrow{O_{m} B_{i}} \times \mathbf{w}$ with ' $\times$ ' denoting the cross product and $\mathbf{w}$ the rotation vector of Euler matrix $R$ given by

$$
\mathbf{w}=\mathbf{K}\left(\begin{array}{c}
\dot{\alpha} \\
\dot{\beta} \\
\dot{\gamma}
\end{array}\right) \text { with } \mathbf{K}=\left(\begin{array}{ccc}
0 & -\sin \alpha & \cos \alpha \cos \beta \\
0 & \cos \alpha & \cos \alpha \cos \beta \\
1 & 0 & -\sin \beta
\end{array}\right)
$$

We thus have

$$
\overrightarrow{\ell_{i}}=\left(\begin{array}{c}
\dot{x} \\
\dot{y} \\
\dot{z}
\end{array}\right)-\overrightarrow{O_{m} B_{i}} \times \mathbf{K}\left(\begin{array}{c}
\dot{\alpha} \\
\dot{\beta} \\
\dot{\gamma}
\end{array}\right)
$$


Finally, as $\overrightarrow{\ell_{i}}=\ell_{i} \overrightarrow{n_{i}}$ one has

$$
\dot{\overrightarrow{\ell_{i}}}=\dot{\ell}_{i} \overrightarrow{n_{i}}+\ell_{i} \dot{\overrightarrow{n_{i}}}
$$

Multiplying both side of the previous equation by $\vec{n}_{i}^{T}$ leads to

$$
\begin{aligned}
\vec{n}_{i}^{T} \dot{\overrightarrow{\ell_{i}}} & =\dot{\ell}_{i} \vec{n}_{i}^{T} \overrightarrow{n_{i}}+l_{i}{\overrightarrow{n_{i}}}^{T} \dot{\overrightarrow{n_{i}}} \\
& =\dot{\ell}_{i}
\end{aligned}
$$

since $\overrightarrow{n_{i}}$ is the unit vector along the $i$-th cable $\left(\vec{n}_{i}^{T} \overrightarrow{n_{i}}=1\right.$ and $\left.\vec{n}_{i}^{T} \overrightarrow{n_{i}}=0\right)$. Using Eqs. (3) and (4), we can write

$$
\dot{\ell}_{i}=\vec{n}_{i}^{T}\left(\begin{array}{c}
\dot{x} \\
\dot{y} \\
\dot{z}
\end{array}\right)-\vec{n}_{i}^{T}\left(\overrightarrow{O_{m} B_{i}} \times \mathbf{K}\left(\begin{array}{c}
\dot{\alpha} \\
\dot{\beta} \\
\dot{\gamma}
\end{array}\right)\right)
$$

Thus, the entries in the Jacobian matrix Eq. (2) are expressed as follows:

$$
\begin{aligned}
\left(\frac{\partial \ell_{i}}{\partial x} \frac{\partial \ell_{i}}{\partial y} \frac{\partial \ell_{i}}{\partial z}\right) & =\vec{n}_{i}^{T} \\
\frac{\partial \ell_{i}}{\partial \alpha} & =-\vec{n}_{i}^{T}\left[\overrightarrow{O_{m} B_{i}} \times\left(\begin{array}{l}
0 \\
0 \\
1
\end{array}\right)\right] \\
\frac{\partial \ell_{i}}{\partial \beta} & =-\vec{n}_{i}^{T}\left[\overrightarrow{O_{m} B_{i}} \times\left(\begin{array}{c}
-\sin (\alpha) \\
\cos (\alpha) \\
0
\end{array}\right)\right] \\
\frac{\partial \ell_{i}}{\partial \gamma} & =-\vec{n}_{i}^{T}\left[\overrightarrow{O_{m} B_{i}} \times\left(\begin{array}{c}
\cos (\alpha) \cos (\beta) \\
\sin (\alpha) \cos (\beta) \\
-\sin (\beta)
\end{array}\right)\right]
\end{aligned}
$$

\section{Forward kinematics and pose estimation}

The forward kinematics problem consists in finding the pose $\mathrm{X}$ of the flying frame for a given cable length vector $\ell=\left(\ell_{1} \cdots \ell_{8}\right)^{T}$, that is, finding $g$ such that

$$
\mathbf{X}=g(\ell)
$$


Each taut cable $i$ defines a spherical workspace of radius $\ell_{i}$ centered at the point $A_{i}$. Finding $g$ is thus related to computing the intersection of the workspace of all taut cables. As there is $a$ priori no analytic expression for $g$, we resort to an optimization technique which involves the (easier) inverse kinematics problem. It consists in minimizing the distance between the cable length vector $\ell_{m}$ measured for the current, yet unknown, pose and the one provided by the inverse kinematics equation $\hat{\ell}=f(\hat{\mathbf{X}})$ for the estimated pose $\hat{\mathbf{X}}$. The error function then can be written as follows:

$$
E=\sum_{i=1}^{8}\left(\ell_{i m}-\hat{\ell}_{i}\right)^{2}
$$

and its gradient with respect to $\hat{\mathrm{X}}$ is:

$$
\nabla E:=\frac{\partial E}{\partial \hat{\mathbf{X}}}=-2 J^{T}\left(\boldsymbol{\ell}_{m}-f(\hat{\mathbf{X}})\right)
$$

The pose is found by gradient descent according to

$$
\hat{\mathbf{X}} \leftarrow \hat{\mathbf{X}}-\eta \nabla E
$$

This pose estimation was implemented in $\mathrm{C}++$ and incorporated in our real time controller. In practice, a few number of iterations (i.e. $n<90$ ) with step size $\eta=0.01$ is sufficient to achieve convergence (i.e. $\nabla E<1 \mathrm{~mm}$ ).

\section{Motor speed command and tension control}

The tension in each cable is estimated using measurements from the winches (actual motor speed $\boldsymbol{v}_{\boldsymbol{m}}$ and motor current $\boldsymbol{I}_{\boldsymbol{m}}$ ) and is corrected using a feed-forward approach. For our DC motors, the following linear relationship holds : $\boldsymbol{I}_{\boldsymbol{m}}=K_{\text {motor }}\left(\boldsymbol{u}-\boldsymbol{v}_{\boldsymbol{m}}\right)$, with $K_{\text {motor }}=-1.646$. The motor speed command, so that $\boldsymbol{v}_{\boldsymbol{m}}=\dot{\boldsymbol{\ell}}$ then writes as $\boldsymbol{u}=\dot{\boldsymbol{\ell}}+\frac{\boldsymbol{I}_{m}}{K_{\text {motor }}}$. Yet, a corrective term $\mathbf{c}=\left(c_{1} \cdots c_{8}\right)^{T}$ is added in order to keep the cable tension within a safe interval, i.e. that prevents cable sagging due to insufficient tension and cable breaking due to excessive tension. 
The motor speed command then becomes $\boldsymbol{u}^{\prime}=\boldsymbol{u}+\boldsymbol{c}$ with $\boldsymbol{c}_{\min } \leq \boldsymbol{c} \leq \boldsymbol{c}_{\max }$. The upper and lower bounds on the corrective term are given by $\boldsymbol{c}_{(\min , \max )}=\frac{\boldsymbol{I}_{(\min , \max )}-\boldsymbol{I}_{m}}{K_{\operatorname{motor}}}+\left(\boldsymbol{v}_{\boldsymbol{m}}-\dot{\boldsymbol{\ell}}\right)$ with $\left(\boldsymbol{I}_{\min }, \boldsymbol{I}_{\max }\right)$ being the admissible range of motor current associated to the safe interval of cable tension. Although several options are possible, we choose to minimize the norm of the corrective term, under the constraint that the correction does not change the pose of the flying frame. The latter can be expressed by considering the static equilibrium equation stating that the additional forces and moments resulting from the corrective term and acting on the flying frame should be zero, that is:

$$
\sum_{i=1}^{8} c_{i} \overrightarrow{n_{i}}=0
$$

and

$$
\sum_{i=1}^{8} c_{i} \overrightarrow{O_{m} B_{i}} \times \overrightarrow{n_{i}}=0
$$

Rewriting the above equations in matrix form leads to the linear constraint $\boldsymbol{A c}=\mathbf{0}_{6 \times 1}$ with

$$
\boldsymbol{A}=\left(\frac{\overrightarrow{n_{1}}}{\overrightarrow{O_{m} B_{1}}} \times \overrightarrow{n_{1}} \cdots \overrightarrow{O_{m} B_{8}} \times \overrightarrow{n_{8}}\right)
$$

Thus the optimization of the corrective term $c$ can be formulated as the following quadratic programming $(\mathrm{QP})$ problem : Min $\boldsymbol{c}^{T} \boldsymbol{c}$ s.t. $\boldsymbol{c}_{\min } \leq \boldsymbol{c} \leq \boldsymbol{c}_{\max }$ and $\boldsymbol{A c}=\mathbf{0}_{6 \times 1}$. The QP problem was solved at each time step (i.e. $10 \mathrm{~ms}$ ) in our real time controller by using a dual active-set method $(2,3)$.

\section{References}

1. A. Pott, Cable-driven parallel robots: theory and application, vol. 120 (Springer, 2018).

2. M. J. Powell, ZQPCVX a FORTRAN subroutine for convex quadratic programming (Department of Applied Mathematics and Theoretical Physics, University, 1983). 
3. D. Goldfarb, A. Idnani, A numerically stable dual method for solving strictly convex quadratic programs., Mathematical Programming 27, 1 (1983). 